\title{
Editorial
}

\section{Advanced Control of Complex Dynamical Systems with Applications}

\author{
Xing-Gang Yan, ${ }^{1}$ Sarah K. Spurgeon, ${ }^{1}$ Qingling Zhang, ${ }^{2}$ and Leonid Fridman ${ }^{3}$ \\ ${ }^{1}$ School of Engineering and Digital Arts, University of Kent, Canterbury CT2 7NT, UK \\ ${ }^{2}$ Institute of Systems Science, Northeastern University, Shenyang, Liaoning 110004, China \\ ${ }^{3}$ Department of Control Engineering, Engineering Faculty, National Autonomous University of Mexico, 58089 Mexico City, Mexico
}

Correspondence should be addressed to Xing-Gang Yan; x.yan@kent.ac.uk

Received 6 March 2016; Accepted 9 March 2016

Copyright (c) 2016 Xing-Gang Yan et al. This is an open access article distributed under the Creative Commons Attribution License, which permits unrestricted use, distribution, and reproduction in any medium, provided the original work is properly cited.

There are many practical systems which can be modelled by dynamical equations. Increasing requirements on system performance mean that within control system analysis and design it is necessary to deal with the increasingly complex dynamical systems that are used to model reality. The complexity stems from nonlinearity, uncertainty, time delay, system singularity, stochastic effects, the presence of interconnections, and so forth. Advances in both technology and control theory have provided the potential to investigate complex dynamical systems. Study on complex systems is becoming increasingly important and this has provided renewed impetus for the development of novel techniques and skills for complex control systems. This provides the motivation for this special issue.

The purpose of this special issue is to provide an opportunity for scientists and control engineers to publish their latest, novel, and original contributions to the development of complex control systems. The special issue focusses on recent achievements and applications in the field of control and analysis for complex systems with a special emphasis on how to solve various control design and/or observer design problems for nonlinear systems and interconnected systems. The considered systems typically involve at least two features such as nonlinearity, disturbances/uncertainty, faults, time delay, interconnections between subsystems, system singularity, stochastic processes, and comparison between simulation and experiments. The special issue focuses on results in theoretical research and practical applications with emphasis on novel methodologies for analysis, design, synthesis, and/or applications. The topics within the special issue include interconnected/networked systems, variable structure control, singular system control, time delay systems, fuzzy control and impulsive control, robust observer design and parameter identification, fault detection and fault tolerant control, analysis and control of stochastic systems, and applications of advanced control systems.

A total of 109 papers were submitted to the special issue for possible publication and 38 of them were accepted and published following a fair and rigorous review process organised by the guest editorial team with the help of the editorial office. The acceptance rate is less than $35 \%$. The accepted papers have been grouped in the following six clusters.

(1) Interconnected/Networked Systems. There are 8 papers considering interconnected or networked systems. The important issue of controllability/observability for interconnected descriptor systems is discussed in "Impulsive Controllability/Observability for Interconnected Descriptor Systems with Two Subsystems" by Q. Zhang et al. Complex networked systems are discussed in the following papers: "Optimal Control of Probabilistic Logic Networks and Its Application to Real-Time Pricing of Electricity" by K. Kobayashi and K. Hiraishi, "Nonfragile Robust $H_{\infty}$ Synchronization Approach for Drive-Response Complex Dynamical Networks with Randomly Occurring Controller Gain Fluctuations and Uncertainties" by D. Li et al., and "Control of Synchronization and Stability for Nonlinear Complex Dynamical Networks 
with Different Dimensional Similar Nodes and Coupling Time-Varying Delay” by L. Yi-ping et al. Decentralised control schemes are proposed in "Decentralized $H_{\infty}$ Control for Large-Scale Systems with Uncertain Missing Measurements Probabilities" by Y. Zhou et al., "Quantized Feedback Control Design of Nonlinear Large-Scale Systems via Decentralized Adaptive Integral Sliding Mode Control," by Y.-M. Xue et al., "Wide-Area Robust Decentralized Coordinated Control of HVDC Power System Based on Polytopic System Theory," by S. Xu et al., and "Time-Varying Scheme for Noncentralized Model Predictive Control of Large-Scale Systems" by A. Núñez et al.

(2) Time Delay System and Sliding Mode Control. Continuous time delay systems are considered in "The Application of Time-Delay Dependent $H_{\infty}$ Control Model in Manufacturing Decision Optimization" by $\mathrm{H}$. Guo et al., and discrete time delay systems are discussed in "Efficient Approach to Stability Analysis of Discrete-Time Systems with TimeVarying Delay" by S. W. Yun and S. H. Kim and "Stabilization of Discrete-Time Delayed Systems via Partially DelayDependent Controllers" by G. Wang and B. Li. Sliding mode techniques are applied in "Terminal Sliding Mode Control with Adaptive Law for Uncertain Nonlinear System” by Z. Zhao et al., "Adaptive Second-Order Sliding Mode Control Design for a Class of Nonlinear Systems with Unknown Input" by Y. Zheng et al., and "Second Order Sliding Mode Control of the Coupled Tanks System" by F. Abu Khadra and J. Abu Qudeiri.

(3) Observers, Filters, and Parameters Identification. An iterative learning control approach with extended observer is proposed to enhance the tracking precision of a telescope in "Iterative Learning Control with Extended State Observer for Telescope System" by H. Cai et al. Different filters are designed for various applications in "Initial Self-Alignment for Marine Rotary SINS Using Novel Adaptive Kalman Filter" by F. Pei et al. and "Model Free Command Filtered Backstepping Control for Marine Power Systems" by $\mathrm{H}$. Zhou et al. Parameter identification is provided in "Channel Parameters Identification Based on IMM Algorithm for Variant Correlation Channel” by Z. Yang et al.

(4) Fuzzy and Impulsive Control. Six papers involve either impulsive control or fuzzy systems. A T-S fuzzy model is considered in "Delay-Dependent Finite-Time $H_{\infty}$ Controller Design for a Kind of Nonlinear Descriptor Systems via a TS Fuzzy Model" by B. Zhu et al. and "A Simplified Output Regulator for a Class of Takagi-Sugeno Fuzzy Models" by T. Hernández-Cortés et al. Control design based on fuzzy techniques is presented in "Fuzzy Logic Based Control of the Lateral Stability of Tractor Semitrailer Vehicle" by X. Yang et al. and "Analytic Model Predictive Control of Uncertain Nonlinear Systems: A Fuzzy Adaptive Approach" by X. Peng et al. Impulsive control is considered in "Impulsive Control of Memristive Chaotic Systems with Impulsive Time Window" by F. Chen et al., and the impulsive CGNNs are considered in "LMI-Based Stability Criterion for Impulsive CGNNs via Fixed Point Theory" by X. Wang et al.

(5) Fault Detection and Fault Tolerant Control. Systems involving faults are considered in "Robust Fault-Tolerant Tracking Control for Nonlinear Networked Control System: Asynchronous Switched Polytopic Approach" by C. Dong et al., "Fault Detection for Multimodal Process Using QualityRelevant Kernel Neighborhood Preserving Embedding” by Y. Fan et al., "FTC with Dynamic Virtual Actuators: Characterization via Dynamic Output Controllers and $H_{\infty}$ Approach" by D. Krokavec et al., and "Defect Detection and Localization of Nonlinear System Based on Particle Filter with an Adaptive Parametric Model" by J. Wu et al.

(6) Other Control and Practical Applications. In this special issue, other approaches such as fractional control, distributed control, backstepping, adaptive control, and model predictive control are also employed by some authors, for example, "Fractional Order Controller Designing with Firefly Algorithm and Parameter Optimization for Hydroturbine Governing System" by L. Junyi and Q. Chen, "Distributed Control Design for Structures Subjected to Traveling Loads" by D. Pisarski, "Adaptive Neural Back-Stepping Control with Constraints for a Flexible Air-Breathing Hypersonic Vehicle" by P. Wang et al., and "Design and Implementation of Recursive Model Predictive Control for Permanent Magnet Synchronous Motor Drives" X. Wu et al. Quite a few papers focus on the application of control methods in various practical systems, for example, "Robust Adaptive Attitude Control for Airbreathing Hypersonic Vehicle with Attitude Constraints and Propulsive Disturbance" by J. Fu et al., "Global Chassis Control System Using Suspension, Steering, and Braking Subsystems" by C. A. Vivas-Lopez et al., "Control Strategy of Three-Phase Photovoltaic Inverter under Low-Voltage Ride-Through Condition" by X. Wang et al., "Research of Control Strategy in the Large Electric Cylinder Position Servo System" by Y. Liu et al., "EndEffector Trajectory Tracking Control of Space Robot with $L_{2}$ Gain Performance" by H. Zhang et al., and "Multiparameter Stochastic Dynamics of Ecological Tourism System with Continuous Visitor Education Interventions" by D. Wei et al.

Although the selected topics and published papers may not represent all of the recent developments in the area, we hope that readers find the special issue helpful and useful.

\section{Acknowledgments}

We sincerely thank all of the reviewers for their valuable comments and suggestions which have contributed greatly to the quality of the special issue.

Xing-Gang Yan Sarah K. Spurgeon

Qingling Zhang

Leonid Fridman 


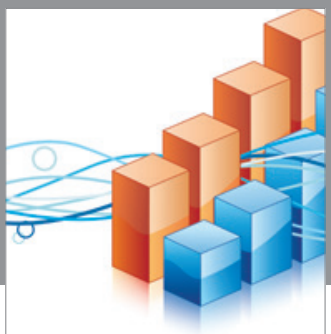

Advances in

Operations Research

vatem alat4

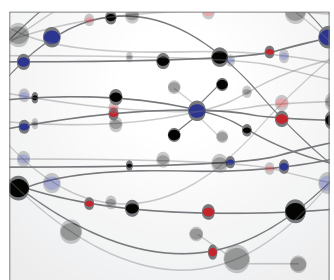

\section{The Scientific} World Journal
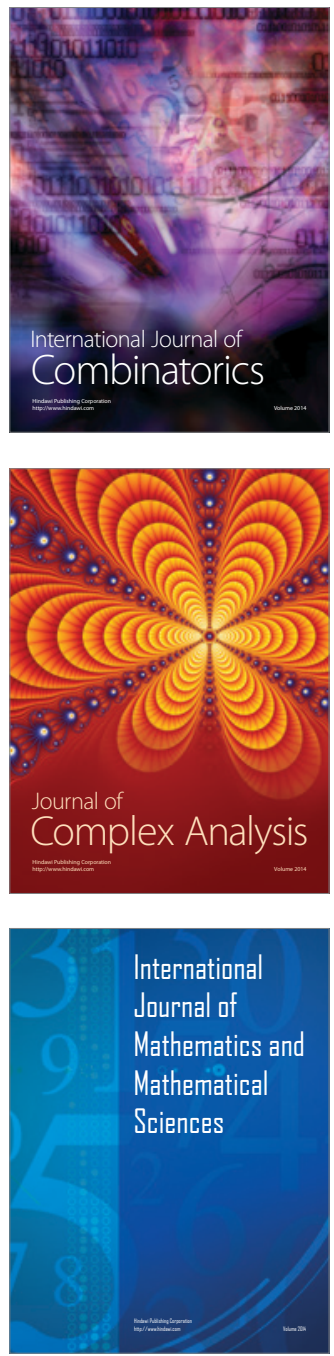
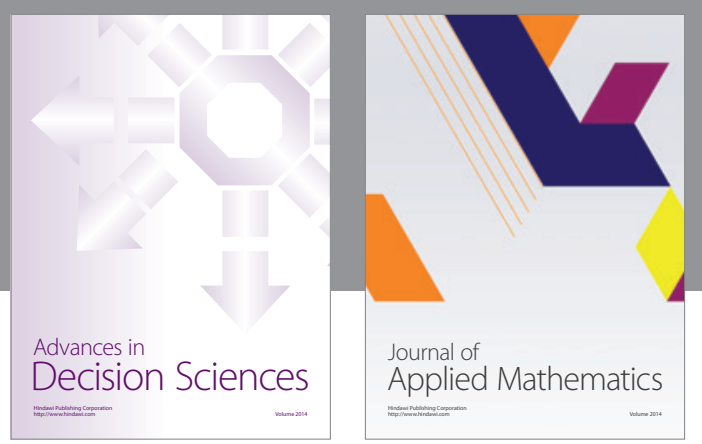

Algebra

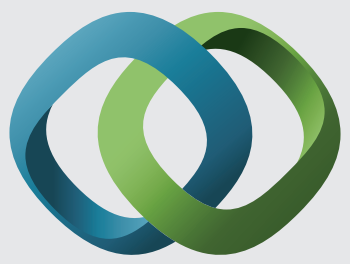

\section{Hindawi}

Submit your manuscripts at

http://www.hindawi.com
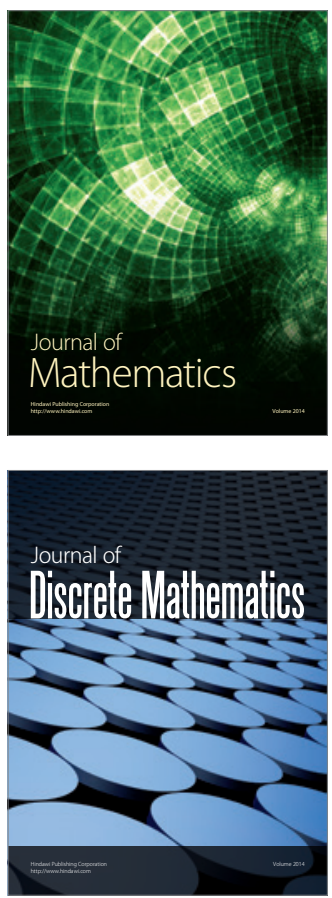

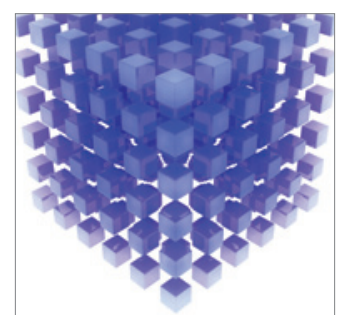

Mathematical Problems in Engineering
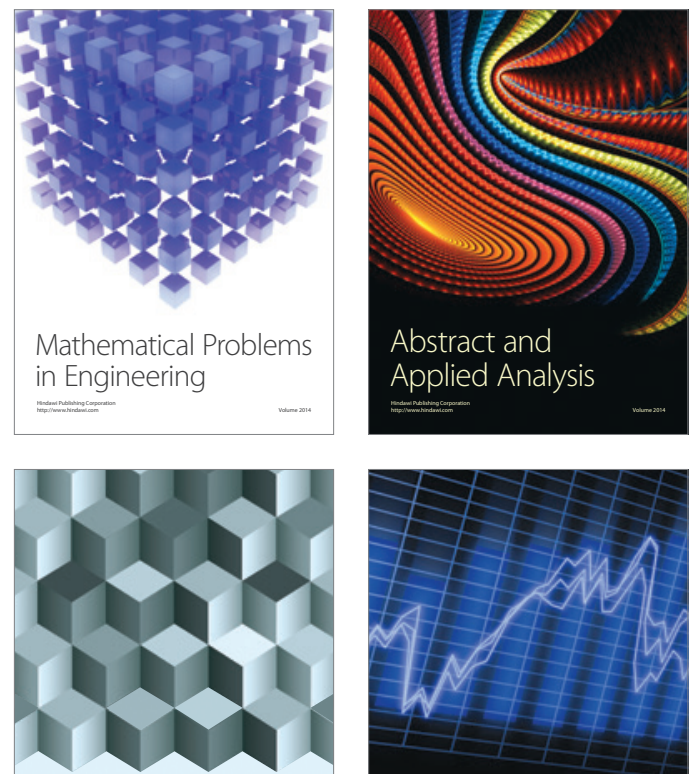

Journal of

Function Spaces

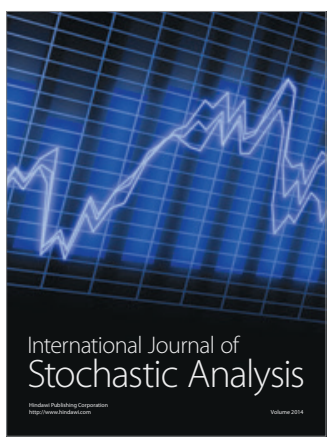

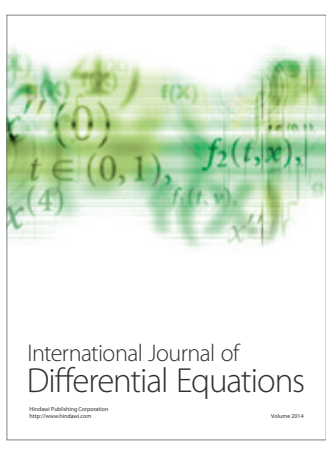
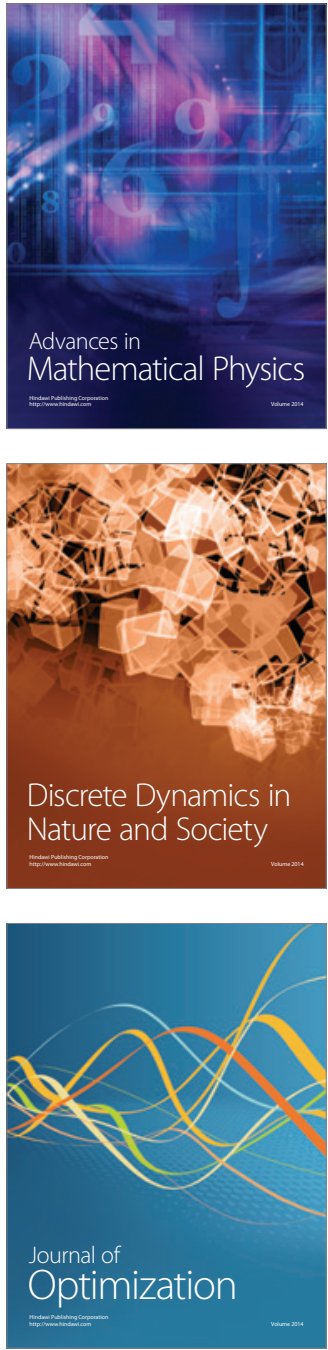